\title{
BMJ Open Impact of removing prescription charges on health outcomes: protocol for a randomised controlled trial
}

Kimberly Cousins (D) , ${ }^{1}$ Pauline Norris, ${ }^{1}$ Simon Horsburgh, ${ }^{2}$ Alesha Smith, ${ }^{3}$
Shirley Keown, ${ }^{4}$ Ariyapala Samaranayaka, ${ }^{5}$ Carlo Marra, ${ }^{3}$ Marianna Churchward ${ }^{6}$

To cite: Cousins $\mathrm{K}$, Norris $\mathrm{P}$, Horsburgh S, et al. Impact of removing prescription charges on health outcomes: protocol for a randomised controlled trial. BMJ Open 2021;11:e049261. doi:10.1136/ bmjopen-2021-049261

- Prepublication history and additional supplemental material for this paper are available online. To view these files, please visit the journal online (http://dx.doi.org/10.1136/ bmjopen-2021-049261)

Received 20 January 2021 Accepted 06 July 2021

Check for updates

(c) Author(s) (or their employer(s)) 2021. Re-use permitted under CC BY-NC. No commercial re-use. See rights and permissions. Published by BMJ.

${ }^{1}$ Centre for Pacific Health, University of Otago Division of Health Sciences, Dunedin, New Zealand

${ }^{2}$ Preventive and Social Medicine, University of Otago-Dunedin Campus, Dunedin, New Zealand ${ }^{3}$ School of Pharmacy, University of Otago Division of Health Sciences, Dunedin, New Zealand ${ }^{4}$ Turanga Health, Gisborne, New Zealand

${ }^{5}$ Centre for Biostatistics, University of Otago Division of Health Sciences, Dunedin, New Zealand

${ }^{6}$ Health Services Research Centre, Victoria University of Wellington, Wellington, New Zealand

Correspondence to Dr Kimberly Cousins; kim.cousins@otago.ac.nz

\section{ABSTRACT}

Introduction Prescription charges prevent many people from accessing the medicines they need to maintain or improve their health. In New Zealand, where most people pay $\$ 5$ per prescription item, Māori and Pacific peoples, those living in most deprived areas and those with chronic health conditions are the most likely to report that cost prevents them from accessing medicines.

Methods and analysis This randomised controlled trial (RCT) will evaluate the effect of removing prescription charges on health outcomes and healthcare utilisation patterns of people with low income and high health needs. We will enrol 2000 participants: half will be allocated to the intervention group and we will pay for their prescription charges for 12 months. The other half will receive usual care. The primary outcome will be hospital bed-days. Secondary outcomes will be: all-cause and diabetes/mental health-specific hospitalisations, prescription medicines dispensed (number and type), deaths, emergency department visits and quality of life as measured by the 5-level EQ-5D version. Costs associated with these outcomes will be compared in an economic substudy. A qualitative substudy will also help understand the impact of free prescriptions on participant well-being using in-depth interviews.

Discussion Being unable to afford prescription medicines is only one of many factors that influence adherence to medicines, but removing prescription charges is relatively simple and in New Zealand would be cheap compared with other policy changes. This RCT will help identify the extent of the impact of a simple intervention to improve access to medicines on health outcomes and health service utilisation.

Ethics and dissemination This study was approved by the Central Health and Disability Ethics Committee (NZ) in July 2019 (19/CEN/33). Findings will be reported in peer-reviewed publications, as well as in professional newsletters, mainstream media and through public meetings.

Trial registration number ACTRN12618001486213p.

\section{INTRODUCTION}

\section{Background}

While the majority of middle-income and high-income countries are able to provide their populations with high-quality health services, not all social groups benefit equally

\section{Strengths and limitations of this study}

This randomised controlled trial is the first to test the effect of eliminating prescription charges on health outcomes, in isolation from other interventions.

- The study targets people who are most likely to experience financial barriers to accessing medicines, including indigenous and ethnic minority populations.

- Unfortunately, there is no centralised method for implementing the intervention, so it relies on pharmacists to provide the intervention.

- Primary care data are not consistently recorded on a national level, so outcomes such as primary care visits cannot be included.

from the services offered. Poorer access to healthcare and negative experiences in the healthcare system can lead to adverse outcomes for some people ${ }^{1}$ and addressing inequalities in health outcomes remains a key issue for many governments.

In New Zealand (NZ), Māori (the country's indigenous population), Pacific people and other minority groups experience barriers to accessing prescription medicines and other care. It has been estimated that if Māori were to receive prescription medicines at the same rate as non-Māori, non-Pacific people with similar burden of disease, Māori would get over 1 million more prescriptions per year, equivalent to a $41 \%$ shortfall in medicines that should have been dispensed..$^{2}$ Less is known about rates of medicine use by Pacific people, but almost a fifth of Pacific adults report prescription charges prevent them from accessing medicines. ${ }^{3}$ There are likely to be many complex reasons for this, which are difficult to address, but there is also a simple, policy-amenable barrier: prescription charges. ${ }^{4}$

As in other high-income countries, the design of the prescription charges regime in NZ and the level of charges have changed 
Box 1 Description of the current prescription charges regime in New Zealand

Medicine cost: New Zealanders pay a variety of charges for their medicines, which cover all or part of the cost of the medicine, dispensing fees and additional fees such as for blister packing. Some medicines are unsubsidised, so patients pay the whole cost plus a dispensing fee; others are partially subsidised so people pay a part charge and a dispensing fee. However, most people pay only a standard $\$ 5$ 'prescription charge' (or copayment) for each prescription item, regardless of the actual cost of the medicine.

Exemptions: There are very few exemptions for the prescription charges. Children under 14 years old are exempted and there are no exemptions based on low income. Some pharmacies absorb the \$5 charge to attract customers (for the pharmacy or supermarket in which they are located). Individuals or families are exempt from prescription charges after they pay for 20 items in one calendar year but there is evidence that most people do not know about this exemption and some continue to be charged the copayment. ${ }^{28}{ }^{29}$ Repeat prescriptions usually do not incur $\$ 5$ prescription charge.

multiple times since they were introduced in the 1980s. A brief overview of current prescription charges in NZ is presented in box 1. Despite the numerous changes, there is little evidence to inform future policy changes. There are strong arguments for keeping prescription charges for the majority of the population, such as reducing waste, encouraging people to value medicines and offsetting the cost of medicines. However, there are also strong arguments that, for some people, prescription charges may lead to significant worsening of health and increased use of other more expensive services, such as publicly funded inpatient hospital care.

Numerous studies have shown that increasing the amount people pay for prescriptions leads to decreased use of prescription medicines. ${ }^{5-7}$ Furthermore, among some patient groups, increased prescription charges lead to poorer health status ${ }^{8}$ and higher use of other healthcare, ${ }^{5910}$ although the quality of evidence is generally low. ${ }^{7}$ Previous research by the authors and others in NZ has shown that people report that prescription charges prevent them from picking up medicines. ${ }^{11}{ }^{12}$ Analysis of Statistics New Zealand's Survey of Family, Income and Employment data suggests that inability to afford prescription medicines is associated with a decline in self-reported health status. ${ }^{13}$ In many countries, such as England and Australia, people on low income or those with chronic conditions pay less than the general population for their prescriptions. While these policies seem to acknowledge that cost is a barrier to accessing medicines for certain groups of people, very few studies have explicitly tested this hypothesis.

Although $\$ 5$ per prescription item is low by international standards, ${ }^{14}{ }^{15}$ there are no exemptions for people on low income and New Zealanders usually also pay for primary care consultations (usually NZ $\$ 15-\mathrm{NZ} \$ 50^{16}$ ); thus, prescription charges are an additional barrier to primary healthcare. As NZ has a high level of income inequality and poverty, ${ }^{18}$ there are many people on very low income for whom prescription charges can be a barrier to accessing medicines. In the latest NZ Health Survey, $5.1 \%$ of the adult population, including $12.7 \%$ of Māori and $14 \%$ of Pacific peoples, reported not picking up at least one medicine in the last 12 months because of cost. Those who lived in the most deprived areas were more than six times as likely to report this as those in the least deprived areas. ${ }^{3}$ The aim of this study is therefore to determine whether removing this barrier (ie, the standard $\$ 5$ prescription charge) leads to decreased use of hospital and other services by people living in areas of high deprivation and with high health needs.

The study will evaluate the effect of removing prescription charges on the usage of publicly funded healthcare services on people with low income and high health needs. It will compare the health outcomes and healthcare utilisation patterns of those who pay standard prescription charges with those who receive free prescriptions. Such an experimental study has not been done in NZ (and very seldom done overseas). If the study shows that removing prescription charges for some groups of people was beneficial, it would be feasible and relatively straightforward to introduce and implement a policy change in NZ.

\section{METHODS AND ANALYSIS}

The Standard Protocol Items: Recommendations for Interventional Trials (SPIRIT) reporting guidelines were used in the development of this study protocol. ${ }^{19}$ The checklist is available as online supplemental file.

\section{Trial design}

The study will employ a two-group parallel randomised controlled trial (RCT) design across three geographical areas, with participants equally randomised to having their prescription charges paid for a year or usual care. On recruitment into the study, participants will complete an interviewer-administered questionnaire covering demographics, health status (including EuroQol-5 Dimension, EQ-5D) and health service use so that the study population can be described and the success of randomisation assessed. Following the 12-month intervention period, hospital bed-days and other secondary outcomes for the groups will be compared between the intervention and control groups.

A diagram depicting the recruitment and data collection process, as provided to participants in the study information sheet, is shown in figure 1 . The SPIRIT diagram is presented in table 1 .

\section{Patient and public involvement}

Prior to undertaking this study, previous qualitative research by PN highlighted the financial barriers many people faced when getting their medicines. A feasibility study was then conducted which aimed to determine the most relevant and appropriate study areas, participant inclusion/exclusion criteria, participant recruitment 


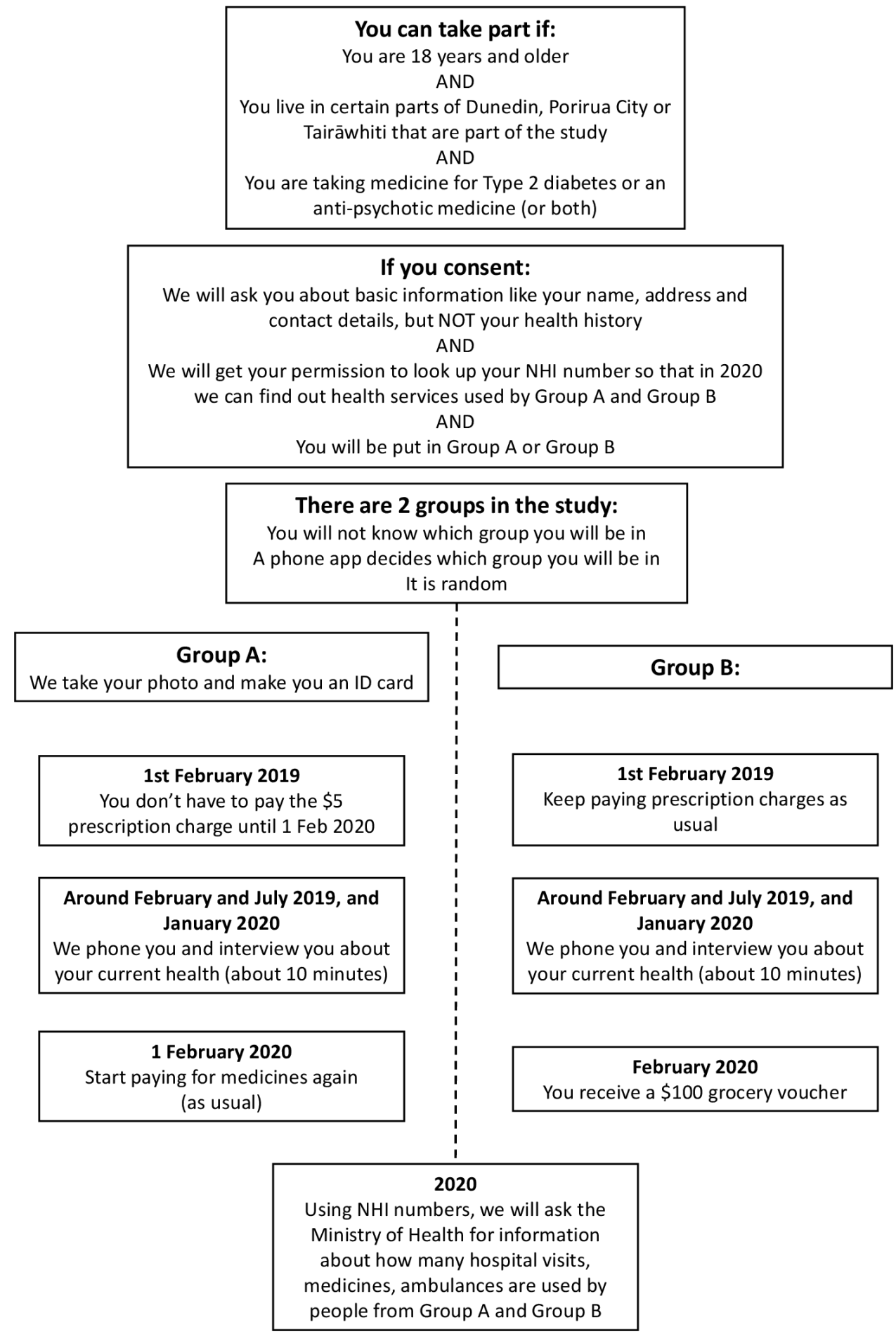

Figure 1 Diagram of inclusion, recruitment and procedure, as provided to all potential participants. NHI, National Health Index.

strategies and prescription payment methods. During the feasibility study, focus groups were conducted with people who resembled our future participants in terms of age, ethnicity and health and social conditions, in order to gain insight into the most acceptable and effective methods of recruiting participants and paying for their medicines. At the beginning of the study, a focus group of people similar to our study participants provided valuable feedback on the participant information sheet in order to make it clearer and easier to understand. Health providers, pharmacists and local community organisations were also consulted during the feasibility study and prior to the beginning of participant recruitment. They are also involved in contacting potential participants and in providing space for recruitment events.

At the end of the study, we will consult local health providers and community organisations again in order to make sure that we disseminate the study findings to participants and their communities in a way that is both culturally appropriate and effective. 
Table 1 SPIRIT diagram for RCT of prescription payment charges: schedule of enrolment, interventions and assessments

\begin{tabular}{|c|c|c|c|c|c|c|}
\hline \multirow[b]{3}{*}{ Timepoint } & \multicolumn{6}{|l|}{ Study period } \\
\hline & \multirow{2}{*}{$\begin{array}{l}\text { Enrolment } \\
\text { October to } \\
\text { December } 2018 \\
-t_{1}\end{array}$} & \multirow{2}{*}{$\begin{array}{l}\text { Allocation } \\
\text { October to } \\
\text { December } 2018 \\
0\end{array}$} & \multicolumn{3}{|c|}{ Postallocation } & \multirow{2}{*}{$\begin{array}{l}\text { Close-out } \\
\text { February to } \\
\text { March } 2020 \\
T_{4} \\
\end{array}$} \\
\hline & & & $\begin{array}{l}\text { February } \\
2019 \\
t_{1}\end{array}$ & $\begin{array}{l}\text { July } \\
2019 \\
t_{2} \\
\end{array}$ & $\begin{array}{l}\text { January } \\
2020 \\
t_{3}\end{array}$ & \\
\hline \multicolumn{7}{|l|}{ Enrolment } \\
\hline Eligibility screen & $\mathrm{X}$ & & & & & \\
\hline ID card (intervention group) & & $x$ & & & & \\
\hline Allocation & & $x$ & & & & \\
\hline \multicolumn{7}{|l|}{ Interventions } \\
\hline Prescription payments & & & $\longleftarrow$ & - & $\rightarrow$ & \\
\hline Hospital bed-days; hospitalisations & & & $\mathrm{x}$ & $x$ & $x$ & $x$ \\
\hline $\begin{array}{l}\text { Dispensing data, other health service } \\
\text { use data }\end{array}$ & & & $x$ & $\mathrm{X}$ & $x$ & $x$ \\
\hline
\end{tabular}

EQ-5D, EuroQol-5 Dimension; RCT, randomised controlled trial; SPIRIT, Standard Protocol Items: Recommendations for Interventional Trials.

\section{Participants}

The study population will be people living in the most deprived areas who have either type 2 diabetes, for which they take medication, or ongoing mental health problems, for which they take antipsychotics. This population has been chosen because individuals are at high risk of not being able to afford their medicines, that is, they are likely to require large numbers of medicines and to have low income. We are aware that people with serious ongoing mental health problems may stop taking their medicines for a range of reasons, one of which is inability to afford them. This study will only determine what difference prescription charges make. Potential participants will be recruited through organisations that serve and/or represent them. The feasibility study that we conducted prior to the study suggested that there are considerable advantages in recruiting people in group settings, and through organisations they know and trust.

\section{Inclusion criteria}

To be eligible for the study, participants must:

- Be at least 18 years old.

- Be able to communicate in English.

- Either have a diagnosis of type 2 diabetes with treatment requiring medication, or taking at least one antipsychotic medication; or both.

- Reside in one of three territorial authorities: Dunedin city, Porirua city or Tairāwhiti district.

- Have a physical address in a census area unit categorised as NZDep 8, 9 or 10. (NZDep is an area-based socioeconomic deprivation score, with NZDep 8, 9 and 10 indicating the most deprivation. ${ }^{20}$ )

\section{Exclusion criteria}

Participants will be excluded if they do not provide a written consent to access prespecified medical information using their unique person identifier for health services, the National Health Index (NHI) number. ${ }^{21}$ Participants will not be excluded if they have other health conditions in addition to those needed to meet the study inclusion criteria.

\section{Study settings}

To ensure ethnic and rural-urban diversity and sufficient numbers of potential participants, the study will be carried out in three geographical regions: Dunedin city, Porirua city and Tairāwhiti district. Dunedin is a mediumsized city with a predominately NZ European population. Porirua is a satellite city of the country's capital, Wellington. It has significant proportions of Māori and Pacific peoples. The Tairāwhiti district is primarily rural, with a predominantly Māori population and a small city (Gisborne). Demographic characteristics of the study sites are presented in table 2.

\section{Intervention}

The intervention group will be exempted from $\$ 5$ prescription charges for 1 year. Ideally, participants would be identified in a national data set, so they were automatically exempt from payment when they received their prescription medicines. Unfortunately, this is impossible 
Table 2 Population, ethnicity and deprivation of study areas

\begin{tabular}{|c|c|c|c|}
\hline & \multicolumn{3}{|c|}{ Territorial authority } \\
\hline & Dunedin city & Porirua city & Gisborne district \\
\hline Census usually resident population (2013) & 120246 & 51717 & 43656 \\
\hline Pacific $(\%)^{\star}$ & $2826(2.4)$ & $12738(24.6)$ & $1539(3.5)$ \\
\hline NZDep2013: 8, 9, 10 census area units & 29862 & 25605 & 28191 \\
\hline Pacific $(\%)^{\star}$ & $1113(3.7)$ & 11064 (43.2) & $1311(4.7)$ \\
\hline
\end{tabular}

Population data retrieved from Statistics New Zealand 2014. 2013 Meshblock data set. ${ }^{30}$

NZDep data retrieved from University of Otago, Wellington. NZDep2013 census area unit data. ${ }^{31}$

*Ethnicity count based on grouped total responses.

so instead participants will be given a photo ID card indicating they are in the study, and pharmacies will be given lists of names and NHI numbers of participants in their geographical region. Pharmacists will be asked to tag participants in their computer systems and to keep the list for use throughout the study. We will visit pharmacies throughout the study period to ensure that the list is still accessible and usable. The Pharmacy Guild of New Zealand, which represents many community pharmacy owners, has also offered to notify its members about the study, in case study participants travel outside their region. The study's free phone number and email address will be supplied to pharmacies and participants to contact us in case of any disputes or confusion. During the feasibility study we trialled this system in Tairāwhiti and it worked well.

We have established good relationships with pharmacies in the study areas and are confident that they will assist with the study. When prescriptions are dispensed for the study participants in the intervention group, the $\$ 5$ charge will be billed to the study. Participants will need to pay for other charges such as blister packing, part charges and the full cost of unsubsidised medicines. This is in line with the likely policy outcome if the study finds a benefit, which is the exemption of certain population groups from the $\$ 5$ charge. Participants and pharmacies will also be instructed that the study funding can only be used to pay for prescriptions dispensed during the study, and not to pay off debt accumulated because of previous charges.

The control group will continue with usual care (usually paying $\$ 5$ for at least the first 20 items they or their family receive). A small number of people in the control group are likely to get some free prescriptions through schemes that provide one-off assistance to people who cannot afford medicines (eg, some primary health organisations provide one-off vouchers). At the end of the study period, control group participants will receive a $\$ 100$ grocery voucher to acknowledge their valuable contribution to the study and to ensure that they do not feel unfairly disadvantaged by their allocation into the control group.

\section{Outcomes}

The primary outcome will be hospital bed-days. Secondary outcomes will be all-cause hospitalisations, prescription medicines dispensed (number and type), hospitalisations for type 2 diabetes/mental health problems, deaths and emergency department visits. We will include all publicly funded hospital and emergency department data (in NZ, all acute care is provided within the public system ${ }^{16}$ ). These will all be accessed through data collections held by the Ministry of Health. Permission to access the data will be requested when we recruit people into the study. Further detail about data access and storage is available from the Australian New Zealand Clinical Trials Registry or from the study authors.

A health economic analysis will be conducted to assess changes in self-reported quality of life, as well as costs associated with hospitalisations. We will administer the 5-level EQ-5D version (EQ-5D-5L) questionnaire for each participant at baseline, at 6 months and at the end of the intervention to calculate quality-adjusted lifeyears (QALYs). We will use the NZ scoring algorithm to assign health state utility values to the EQ-5D-5L health vectors as per the national Pharmaceutical Management Agency (PHARMAC, the government body responsible for funding medicines) recommendations.

We will also do a qualitative substudy of a small number of participants in the intervention group, to record and explore their experiences, and the impact of the intervention on their daily lives and expenditure. Data will be collected using in-depth phone interviews with participants who were either selected randomly or were identified at recruitment as good candidates for the qualitative substudy based on their health and living situation.

\section{Study duration}

The intervention will last for 12 months from 1 February 2019 to 31 January 2020. This timeframe has been chosen because it aligns with the government's own prescription calendar year: all New Zealanders must pay prescription charges from 1 February each year until they have paid for 20 prescriptions, after which they no longer have to 
pay the charges, that is, the prescription count 'resets' on 1 February. Previous studies have found that impacts of copayments are quickly evident. Tamblyn et al found significantly increased adverse events including hospitalisations in a 10-month period following an increase in copayments in Canada. ${ }^{9}$ Previous studies have found that non-adherence to diabetes medication is associated with significantly increased hospitalisations. ${ }^{22-24}$ These studies measured adherence using a medication possession ratio calculated over 1 year, and hospitalisations in the subsequent year, suggesting that the impact is relatively rapid. $\mathrm{Lau}$ and $\mathrm{Nau}^{24}$ did not include people taking insulin, where presumably the effect may be even stronger and quicker.

\section{Sample size}

Data from the NZ Atlas of Healthcare Variation suggest that on average people with diabetes in our study regions spend 1.61 days/year in hospital. ${ }^{25}$ This is likely to underestimate the number of bed-days for people with type 2 diabetes in our study population, who live in areas of high deprivation and are therefore likely to have more comorbidities and have less well-managed diabetes than average, leading to high hospitalisation rates. People taking antipsychotics have high incidence of hospitalisation (our unpublished analyses suggest that 38\% of people taking antipsychotics were hospitalised in a year) and hospitalisations for mental health tend to be long, with an average stay of 17.4 days. ${ }^{26}$ Therefore, the number of bed-days per person for people living in highly deprived areas and taking antipsychotics is unlikely to be lower than the figure for people with diabetes. Assuming the rate of 1.61 bed-days per person, a sample size of 943 in each group would give us $80 \%$ power to detect a $10 \%$ reduction in bed-days in the intervention group. We aim to recruit 1000 people in each group to allow for people who choose to withdraw from the study.

\section{Randomisation (sequence generation and type)}

People who consent to participate will be randomly allocated to the intervention or control group at recruitment in a ratio of $1: 1$, with no blocking or stratification on geographical location, and with the allocation sequence concealed from the recruiter and from EQ-5D interviewers (at 6 and 12-month interviews).

\section{Statistical methods}

The study population will be described and baseline characteristics of the two groups will be compared using descriptive statistical techniques. The primary outcome and each of the other outcomes measured as counts will be assessed using a suitable version of regression model in the Poisson family to compare rates between two groups during the follow-up period (with 95\% CIs) while allowing for control of any unbalanced baseline characteristics. We will follow an intention-to-treat analysis, that is, all participants will be analysed in their allocated groups regardless of whether their prescription charges were paid for by the study or by other sources.

We will perform a cost-effectiveness analysis with the incremental cost per QALY calculated from the health systems perspective. The time horizon for the economic analysis will be for the duration of the trial and modelled out for 10 years. Participants who are not contactable, unable or unwilling to complete the EQ-5D questionnaire at all three timepoints (baseline, 6 months, 12 months) will be excluded from the QALY analyses. Qualitative data from in-depth interviews will be analysed thematically.

\section{DISCUSSION}

Access to medicines is a human right, and poor access is likely to be bad for people's health, and may increase costs to the health system. Being unable to afford prescription medicines is only one of many factors that influence adherence to medicines. Many of these, such as patient concerns about medicines, are complex and multifactorial. ${ }^{27}$

Conversely, prescription charges can be changed or removed relatively simply, and in NZ they are low and so could be removed at modest cost. The strength of an RCT is that it isolates one factor, changes this for the intervention group, but not for the control group, and measures the impact. Thus, we modelled the intervention on what is likely to happen if the government, local health authorities or others chose to exempt people with low income and/or high health needs from prescription charges.

It is possible that covering the $\$ 5$ prescription charge may be insufficient to improve the health outcomes and reduce the burden on the health system of these particularly vulnerable population groups. Many patients incur additional costs when collecting prescriptions, such as for blister packing and for medicines that are not fully subsidised. We did not cover these additional charges in our trial because it was unlikely that these additional costs would be covered by a policy change to address prescription charges: blister packing charges are set by individual pharmacies and medicine subsidies are determined by PHARMAC, the government's medicine funding agency.

Over the past few decades in many countries, prescription copayments have increased and the direct cost of prescription medicines has shifted from the health system or insurer to the individual. This occurred in $\mathrm{NZ}$ when the copayment increased from $\$ 3$ to $\$ 5$ in 2013 . However, the shift of the cost burden away from the health provider/ insurer is only an advantage to the health system if it does not lead to increases in costs of providing other services. Our hypothesis is that the cost of fully covering prescription charges for poor, chronically unhealthy individuals pales in comparison to the costs associated with their subsequent decrease in health status resulting from their inability to pay for medicines. The perceived health 'savings' do not consider the health system as a whole.

No other studies have used an RCT design to investigate and measure the extent of the impact of removing 
prescription charges for those with low socioeconomic status and high health needs. Thus, the findings from this study will inform current and future governments in NZ and elsewhere. By using a multifaceted analytical approach, we will generate sound scientific evidence to determine the impact of exempting these groups from prescription charges on individuals' health and wellbeing outcomes, health service utilisation and costs to the health system.

\section{ETHICS AND DISSEMINATION}

\section{Ethics approval and consent to participate}

Ethics approval for this study was granted by the Central Health and Disability Ethics Committee of NZ in July 2019 (19/CEN/33).

All potential participants will be given an information sheet describing what the study is about and what is involved if they choose to participate, and written consent will be required in order for them to take part (these are available on request). Members of the research team will be available to answer questions about the study at the time of recruitment and all participants will be given the research team's free phone number and email address, should they have any questions during the study.

\section{Safety considerations}

Unlike most RCTs, we do not expect the intervention used in this study to be associated with unforeseeable negative side effects, though participants will be encouraged to speak to their health providers if they plan to restart taking medicines because of the study. For this reason, there will not be a data monitoring team and interim analyses will not be conducted during the intervention period.

\section{Dissemination of study findings}

The study findings will be presented at local and international conferences and submitted for publication in relevant peer-reviewed academic journals. In addition, we will consult with local health providers and community organisations to determine the most appropriate and effective way of sharing the findings in the wider community in each study area. We also plan to approach news media outlets (both local and national) to disseminate the study findings more widely to the general public, following peer review and publication in academic journals. A final report of the study findings will be disseminated to local health boards, pharmacy organisations and other relevant stakeholders and policymakers.

Contributors $\mathrm{PN}$ is the principal investigator and conceived the study. A feasibility study was carried out by PN, KC, SK, ASm and SH. PN and KC designed the study and drafted the study protocol, with input from ASm, ASa, CM, SK, SH and MC. ASa is the biostatistician on the study and assisted with overall design and sample size calculations. CM provided detail for the health economic aspects of the study. SH and ASm provided detail for the health outcome data. MC and SK provided study site-specific advice on participant recruitment and Māori and Pacific input into the study. All authors read and approved the final manuscript.
Funding The study is funded by the Health Research Council of New Zealand (HRC Ref 18/134). The cost of the prescription charges for the study is cofunded by the Pharmaceutical Management Agency (PHARMAC), the New Zealand Crown-owned entity that determines which medicines will be funded in New Zealand.

Competing interests None declared.

Patient consent for publication Not required.

Provenance and peer review Not commissioned; externally peer reviewed.

Supplemental material This content has been supplied by the author(s). It has not been vetted by BMJ Publishing Group Limited (BMJ) and may not have been peer-reviewed. Any opinions or recommendations discussed are solely those of the author(s) and are not endorsed by BMJ. BMJ disclaims all liability and responsibility arising from any reliance placed on the content. Where the content includes any translated material, BMJ does not warrant the accuracy and reliability of the translations (including but not limited to local regulations, clinical guidelines, terminology, drug names and drug dosages), and is not responsible for any error and/or omissions arising from translation and adaptation or otherwise.

Open access This is an open access article distributed in accordance with the Creative Commons Attribution Non Commercial (CC BY-NC 4.0) license, which permits others to distribute, remix, adapt, build upon this work non-commercially, and license their derivative works on different terms, provided the original work is properly cited, appropriate credit is given, any changes made indicated, and the use is non-commercial. See: http://creativecommons.org/licenses/by-nc/4.0/.

ORCID iD

Kimberly Cousins http://orcid.org/0000-0002-3449-8295

\section{REFERENCES}

1 Robson B, Purdie G, Cormack D. Unequal impact: Māori and nonMāori cancer statistics 1996-2001. Wellington: Ministry of Health, 2006.

2 Metcalfe S, Beyene K, Urlich J, et al. Te Wero tonu-the challenge continues: Māori access to medicines 2006/07-2012/13 update. NZ Med J 2018;131:27-47.

3 Ministry of Health. Annual update of key results 2019/20: New Zealand Health Survey. Wellington Ministry of Health; 2020.

4 PHARMAC. Achieving medicine access equity in Aotearoa New Zealand: towards a theory of change: Pharmac, 2019.

5 Goldman DP, Joyce GF, Zheng Y. Prescription drug cost sharing: associations with medication and medical utilization and spending and health. JAMA 2007;298:61-9.

6 Hynd A, Roughead EE, Preen DB, et al. The impact of co-payment increases on dispensings of government-subsidised medicines in Australia. Pharmacoepidemiol Drug Saf 2008;17:1091-9.

7 Luiza VL, Chaves LA, Silva RM, et al. Pharmaceutical policies: effects of cap and co-payment on rational use of medicines. Cochrane Database Syst Rev 2015:CD007017.

8 Heisler M, Langa KM, Eby EL, et al. The health effects of restricting prescription medication use because of cost. Med Care 2004;42:626-34.

9 Tamblyn R, Laprise R, Hanley JA, et al. Adverse events associated with prescription drug cost-sharing among poor and elderly persons. JAMA 2001;285:421-9.

10 Mojtabai R, Olfson M. Medication costs, adherence, and health outcomes among Medicare beneficiaries. Health Aff 2003;22:220-9.

11 Barnett R. "Wait till it's serious:" health care costs and urban survival strategies of low income groups in Christchurch. N Z Med J 2000;113:350-4.

12 Norris $\mathrm{P}$, Tordoff $\mathrm{J}$, Mclntosh B, et al. Impact of prescription charges on people living in poverty: a qualitative study. Res Social Adm Pharm 2016;12:893-902.

13 Jatrana S, Richardson K, Norris P, et al. Is cost-related non-collection of prescriptions associated with a reduction in health? findings from a large-scale longitudinal study of New Zealand adults. BMJ Open 2015;5:e007781.

14 Australian Government Department of Health. About the PBS. Available: http://www.pbs.gov.au/info/about-the-pbs [Accessed 16 Sep 2014].

15 Kanavos P, Vandoros S, Irwin R. Differences in costs of and access to pharmaceutical products in the EU, 2010.

16 Gauld R. New Zealand Overview. In: Tikkanen R, Osborn R, Mossialos E, eds. International health care system profiles. Online: The Commonwealth Fund, 2020. 
17 Quandl. Gini Index By Country [updated 27 March 2014]. Available: http://www.quandl.com/demography/gini-index-by-country [Accessed 17 Apr 2014].

18 Bellamy P. Household incomes, inequality and poverty Wellington: New Zealand Parliament, 2011. Available: http://www.parliament. nz/mi-nz/parl-support/research-papers/00PlibCIP181/householdincomes-inequality-and-poverty [Accessed 17 Apr 2014].

19 Chan A-W, Tetzlaff JM, Gøtzsche PC, et al. SPIRIT 2013 explanation and elaboration: guidance for protocols of clinical trials. BMJ 2013;346:e7586.

20 Atkinson J, Salmond C, Crampton P. NZDep2013 Index of Deprivation. Wellington Department of Public Health, University of Otago, Wellington; 2014.

21 Ministry of Health. National Health Index Online: Ministry of Health; [updated 17 Dec 2020]. Available: https://www.health.govt.nz/ our-work/health-identity/national-health-index [Accessed 17 Dec 2020].

22 Lo-Ciganic W-H, Donohue JM, Jones BL, et al. Trajectories of diabetes medication adherence and hospitalization risk: a retrospective cohort study in a large state Medicaid program. J Gen Intern Med 2016;31:1052-60.

23 Ho PM, Rumsfeld JS, Masoudi FA. Effect of medication nonadherence on hospitalization and mortality among patients with diabetes mellitus. Arch Intern Med 2006;166:1836-41.
24 Lau DT, Nau DP. Oral antihyperglycemic medication nonadherence and subsequent hospitalization among individuals with type 2 diabetes. Diabetes Care 2004;27:2149-53.

25 Health Quality and Safety Commission New Zealand. Atlas of healthcare Variation|Diabetes management. Medico-surgical beddays for people with diabetes, 2015. Available: http://www.hqsc. govt.nz/our-programmes/health-quality-evaluation/projects/atlas-ofhealthcare-variation/ [Accessed 30 Jun 2017].

26 Health Quality and Safety Commission New Zealand. HQSC atlas: mental health key performance indicators. Wellington: Health Quality and Safety Commission New Zealand. Available: https://www.hqsc. govt.nz/assets/Health-Quality-Evaluation/Atlas/MentalHealthSF9Dec/ atlas.html [Accessed 7 Jul 2017]

27 Pound P, Britten N, Morgan M, et al. Resisting medicines: a synthesis of qualitative studies of medicine taking. Soc Sci Med 2005;61:133-55.

28 Norris P, Horsburgh S, Cumming J. Press release: Kiwis missing out on free prescription medicines entitlement, 2012.

29 Norris PT, Wilson SE, Green JA, et al. Knowledge and attitudes to prescription charges in New Zealand and England. Res Social Adm Pharm 2018;14:180-6.

30 Ministry of Health. 2013 Meshblock dataset. Wellington: Statistics New Zealand, 2014.

31 University of Otago Wellington. NZDep2013 census area unit data. University of Otago Wellington, 2014. 